\title{
A NOTE ON THE DIRICHLET PROBLEM AT INFINITY FOR MANIFOLDS OF NEGATIVE CURVATURE
}

\author{
ALBERT BORBÉLY
}

(Communicated by Jonathan M. Rosenberg)

\begin{abstract}
M. T. Anderson and D. Sullivan showed that the Dirichlet problem at infinity for simply connected manifolds is solvable if the curvature satisfies $-a^{2}<K<-b^{2}$. Using M. T. Anderson's method we generalize this statement to manifolds satisfying the weaker bounds $-g(r)<K<-b^{2}$, where $g(r) \approx$ $e^{\lambda r}$, with $\lambda<1 / 3$.
\end{abstract}

\section{INTRODUCTION}

This paper is concerned with the solution of the Dirichlet problem at infinity.

We refer to [1] for the background of this problem. There are two different approaches to the question. The first one uses probabilistic methods like $[8,6$, 2]. The second one is more geometric and is based upon constructing convex sets with nontrivial behavior at infinity. It was H. I. Choi [4] who showed that the Dirichlet problem can be solved if the curvature is bounded away from zero and every two points at infinity can be separated by convex sets. Namely he proved the following theorem in [4]. We refer to $\S 1$ for the definition of $S^{n-1}(\infty)$, the sphere at infinity.

Theorem A. Let $N^{n}$ be a complete simply connected Riemannian manifold with sectional curvature $K$, satisfying $K \leq-1$ and suppose for any distinct $x, y \in$ $S^{n-1}(\infty)$ that there exist disjoint open neighborhoods $V_{x}, V_{y}$ of $x, y$ in $\bar{N}^{n}=$ $N^{n} \cup S^{n-1}(\infty)$ such that $V_{x} \cap N^{n}$ and $V_{y} \cap N^{n}$ are strictly convex. Then the Dirichlet problem at infinity for $\Delta$ is uniquely solvable for any $f \in C^{0}\left(S^{n-1}(\infty)\right)$.

M. T. Anderson in [1] managed to construct appropriate convex sets under the assumption that the curvature satisfies $-a^{2}<K<-b^{2}$, for some constants $a, b>0$. His theorem, which is the same as D. Sullivan's [8] is the following:

Theorem B. Let $N^{n}$ be a complete simply connected Riemannian manifold with sectional curvature $K$, satisfying $-a^{2}<K<-b^{2}$, for some constants $a, b>$ 0 . Then the Dirichlet problem at infinity for $\Delta$ is uniquely solvable for any $f \in C^{0}\left(S^{n-1}(\infty)\right)$.

Received by the editors July 5, 1990 and, in revised form, September 10, 1990.

1980 Mathematics Subject Classification (1985 Revision). Primary 58G20; Secondary 53C20.

Key words and phrases. Dirichlet problem, harmonic functions. 
With a modification of his argument we can replace the lower bound of the curvature with a growth condition to obtain the following:

Theorem. 0.1. Let $N^{n}$ be a complete simply connected Riemannian manifold with sectional curvature $K$, and $O \in N^{n}$ fixed. Denote by $g(r)$ the absolute value of the minimal sectional curvature in the closed ball of radius $r$ about $O$. Suppose the following two conditions hold:

(i) $K \leq-1$,

(ii) $g(r)<e^{\lambda r}$ if $r>r_{0}$, for some $\lambda<1 / 3$.

Then the Dirichlet problem at infinity for $\Delta$ is uniquely solvable for any $f \in$ $C^{0}\left(S^{n-1}(\infty)\right)$.

Remark. It is obvious, of course, that if there is an $O \in N^{n}$ satisfying (ii) then every point of $N^{n}$ will satisfy the same condition.

\section{Preliminaries}

Because our proof is based upon that of M. T. Anderson we follow his notation closely. So let $N^{n}$ be a complete simply connected Riemannian manifold with sectional curvature $K$ satisfying $K \leq-1$. We denote the sphere at infinity by $S^{n-1}(\infty)$ which is the set of asymptote classes of geodesic rays; two rays $\gamma_{1}, \gamma_{2}:[0, \infty) \rightarrow N^{n}$ define the same asymptote class if $\lim _{t \rightarrow \infty} \operatorname{dist}\left(\gamma_{1}(t), \gamma_{2}(t)\right)$ $<\infty$. There is a natural topology on $\bar{N}^{n}=N^{n} \cup S^{n-1}(\infty)$, called the cone topology which is defined in [5] (see also in [1]). Let $O \in N^{n}$ be arbitrary point, $v \in T_{O} N^{n}$ and denote the truncated cone around $v$ by

$$
T(v, \alpha, r)=\left\{P \in N^{n}: \angle(v, \overrightarrow{O P})<\alpha, \operatorname{dist}(O, P)>r\right\},
$$

where $0<\alpha<\pi, r \geq 0$, and $\angle$ denotes the angle in $T N^{n}$. We denote the cone around $v$ by $T(v, \alpha)=T(v, \alpha, 0)$. The truncated cones together with geodesic balls give a basis for the cone topology of $\bar{N}^{n}$. Eberlein and O'Neill [5] have shown that it does not depend on the choice of $O$.

$S_{O}(r)$, (resp. $B_{O}(r)$ ) denotes the geodesic sphere (resp. the closed geodesic ball) of radius $r$ centered around $O$. $\rho_{O}$ denotes the distance function from $O$.

Construction of convex sets. Let $P \in N^{n}$ be fixed. In this section we construct a convex set that contains some cone centered at $P$ but does not contain some truncated cone with vertex $P$. These convex sets will satisfy the conditions in Choi's theorem (Theorem A) yielding Theorem 0.1. Our construction is the same as Anderson's with the exception of some slight modification. His construction was based upon the fact that if the curvature $K<-1$ then the 2nd fundamental form $U$ of $S(r)=S_{O}(r)$ remains "big" even when $r$ is large i.e. $U>\mathbf{I}$, where $\mathbf{I}$ is the identity (i.e. $\left.\langle U(X), X\rangle>|X|^{2}\right)$. Then we can scoop out a small piece such that the remaining truncated ball is still convex. Now repeating this process, taking bigger balls and scooping out more and more pieces he shows that the union of these truncated balls in some direction contains a cone and in some other direction it does not contain a truncated cone. Everything depends on the relationship between the diameter of the scoop and the "depth" of the scoop. That is where he uses the lower bound on the curvature.

We will let the sectional curvature go to $-\infty$ but using the growth condition we will still be able to get the right estimates. 
We start with the analog of [1, Proposition 2.2].

Proposition 1.1. Let $P \in N^{n}$ and $Q \in S_{P}(r)$ for some $r$. Define " $a$ " by the following formula:

$$
a=\max \left\{|K(\alpha)|: \alpha \text { is a two - plane at } R \in B_{P}(r+1)\right\} \text {. }
$$

Then there is $a \delta>0$ absolute constant and a function $f \in C^{\infty}\left(N^{n}\right), 0 \leq f \leq$ 1, $f(Q)=0$, and $f \equiv 1$ outside $B_{Q}(1)$ such that the sublevel sets of $\rho_{P}-\varepsilon f$ are strictly convex subsets of $N^{n}$, where $\varepsilon=\delta / a$.

The proof is the same as of [1, Proposition 2.2] but for the sake of completeness we include it.

Proof. Let $\varphi$ be a fixed "good" approximation of $\min \{\max \{0,2 t-1\}, 1\}$ such that $\varphi \equiv 0$ on $[0,1 / 2]$ and $\varphi \equiv 1$ on $[1, \infty]$. Now there is a constant $L$ such that $\varphi^{\prime}<L$ and $\varphi^{\prime \prime}<L$. Let $f=\varphi\left(\rho_{Q}\right)$. Then $D^{2} f=\varphi^{\prime \prime} \cdot d \rho \otimes d \rho+\varphi^{\prime} \cdot D^{2} \rho$ (where $D^{2} f$ denotes the Hessian of $f$ ).

Using the comparison theorem, we have that for all $R \in B_{Q}(1)$

$$
\left|D^{2} \rho_{P}(R)\right|<a \cdot \operatorname{coth}\left(a \cdot \rho_{Q}(R)\right) .
$$

From the construction of $\varphi$ it is immediate that

$$
\left|D^{2} f\right|<L+L \cdot a \cdot \operatorname{coth}(1 / 2) \text {. }
$$

We now compute the 2 nd fundamental form of $\left\{\rho_{P}-\varepsilon f=r\right\}$.

$$
\| \rho-\varepsilon f(X, X)=\frac{1}{|d \rho-\varepsilon d f|} \cdot\left(D^{2} \rho-\varepsilon \cdot D^{2} f\right)(X, X)
$$

for tangent vectors $X$ satisfying $d\left(\rho_{P}-\varepsilon f\right)(X)=0$. Because $d f=\varphi^{\prime} d \rho_{Q}$ and $\varphi^{\prime}<L$, this implies that if $\varepsilon$ is sufficiently small then $X$ is almost tangential to the sphere around $P$. On the other hand $D^{2} \rho_{P}(Y, Y)>1$ for $d \rho_{P}(Y)=0$, $|Y|=1$ (because $K_{N} \leq-1$ ), so if we choose $\delta$ small enough then $\|_{\rho-\varepsilon f}$ will be positive definite. Hence Proposition 1.1 is proved.

We now start constructing a convex set outside some truncated cone exactly the same way as M. T. Anderson.

Let $v \in T_{P} N^{n}$ and $r=r_{0}>0$. Pick some $\alpha$, satisfying $0<\alpha<\pi$. We write $B(r)=B_{P}(r)$ and $S(r)=S_{P}(r)$.

We now define different sets by induction:

$$
C_{0}=B(r), \quad T_{0}=\{Q \in S(r): \angle(v, \overrightarrow{O Q})<\alpha\}, \quad D_{0}=\varnothing .
$$

For each $Q \in T_{0}$ choose the function $f$ and the number $\varepsilon_{0}$ according to Proposition 1.1. Because " $a$ " in Proposition 1.1 does not depend on the choice of $Q, \varepsilon_{0}$ is independent of $Q$. The function $f$ will of course depend on $Q$. In the next step we define

$$
\begin{gathered}
C_{1, Q}=\left\{R \in N^{n}: \rho_{P}-\varepsilon_{0} f \leq r_{0}\right\}, \quad \widetilde{C}_{1}=\bigcap\left\{C_{1, Q}: Q \in T_{0}\right\}, \\
C_{1}=\widetilde{C}_{1} \backslash D_{0}, \quad D_{1}=B\left(r_{1}\right) \backslash C_{1}, \quad T_{1}=\overline{S\left(r_{1}\right) \backslash \partial C_{1}}, \quad \text { and } \quad r_{1}=r_{0}+\varepsilon_{0} .
\end{gathered}
$$

Similarly for each $Q \in T_{1}$ we choose $f$ and $\varepsilon_{1}$, where $\varepsilon_{1}$ is independent of $Q$ and construct

$$
C_{2, Q}=\left\{R \in N^{n}: \rho_{P}-\varepsilon_{1} f \leq r_{1}\right\}, \quad \widetilde{C}_{2}=\bigcap\left\{C_{2, Q}: Q \in T_{1}\right\},
$$




$$
C_{2}=\widetilde{C}_{2} \backslash D_{1}, \quad D_{2}=B\left(r_{2}\right) \backslash C_{2}, \quad T_{2}=\overline{S\left(r_{2}\right) \backslash \partial C_{2}}, \quad \text { and } \quad r_{2}=r_{1}+\varepsilon_{1} .
$$

Now it is clear how to proceed inductively. We have to prove that the $C_{i}$ 's are convex sets. The argument of [1] works here too. Proposition 1.1. implies that $C_{1}$ and $\widetilde{C}_{2}$ are convex, because these are intersections of convex sets. Suppose $X, Y \in \partial C_{2}$ such that the geodesic $\gamma$ joining $X$ and $Y$ enters $D_{2}$. Because $\widetilde{C}_{2}$ is convex $\gamma \in \widetilde{C}_{2}$, so it must enter $D_{1}$. The convexity of $C_{1}$ implies that $\gamma$ must intersect $\partial D_{1} \backslash \partial C_{1}=\operatorname{int}\left(T_{1}\right)$, which contradicts $\gamma \subset \widetilde{C}_{2}$. This proves the convexity of $C_{2}$ and the same argument applies to $C_{n}$. Let $C=C(v, \alpha, r)=\bigcup C_{n}$, the desired convex set.

Behavior at infinity. It is obvious that $C(v, \alpha, r) \cap T(v, \alpha, r+\eta)=\varnothing$ for any $\eta>0$ because if a geodesic ray $\gamma$ from $P$ leaves $C$ through $T_{0}$ the convexity of $C$ prevents $\gamma$ ever intersecting $C$ again. Hence the closure of $C$ misses an open set of $S^{n-1}(\infty)$.

To prove that $\bar{C}$ contains an open set of $S^{n-1}(\infty)$ it is useful to have some estimate on the viewing angle of balls.

Lemma 1.2. Let $N^{n}$ be a complete, simply connected Riemannian manifold with sectional curvature $K \leq-1$. Let $O, P \in N^{n}$, $\operatorname{dist}(O, P)=r$, and $r>b>0$. Denote by $\varphi$ the half-viewing angle of the sphere $S_{P}(b)$ from $O$, that is $\varphi=$ $\max \left\{\angle(O P, O Q): Q \in S_{P}(b)\right\}$. Then we have

$$
\varphi<b e^{-(r-b)} / 2 \text {. }
$$

Proof. We use comparison theorems to show that the viewing angle in $N^{n}$ is smaller than the corresponding viewing angle in the hyperbolic space $H^{2}(-1)$.

Let $Q \in S_{P}(b)$ be such that the angle $\angle(O P, O Q)$ is maximal. Now look at the corresponding geodesic triangle $\widetilde{O} \widetilde{P} \widetilde{Q}$ in $H^{2}(-1)$, such that the corresponding sides have the same length. Topogonov's theorem [7, Theorem 2.7.6] tells us that

$$
\varphi=\angle(O P, O Q) \leq \angle(\widetilde{O P}, \tilde{O} \widetilde{Q}) \leq \tilde{\varphi},
$$

where $\tilde{\varphi}$ is the half-viewing angle of the ball $B_{\widetilde{P}}(b)$ in $H^{2}(-1)$. So it is enough to estimate the viewing angle in $H^{2}(-1)$. We use the Poincaré model and because everything takes place in $H^{2}(-1)$ we do not use " " for the rest of the proof.

Recall that the hyperbolic (metric) on the Euclidean ball $B^{2}(1)$ is given by

$$
d s_{H}=\frac{2}{1-t^{2}} \cdot d s_{E}
$$

where $d s_{E}$ is the Euclidean distance.

Let $O$ denote the center of $B^{2}(1)$ and let $P \in B^{2}(1)$ with $\operatorname{dist}_{H}(O, P)=$ $r$. The geodesic sphere $S_{P}(b)$ intersects the line segment $O P$ in $X$. Then $\operatorname{dist}_{H}(O, X)=r-b$. From (1.4) the Euclidean distance of $O X$ can be easily computed:

$$
\operatorname{dist}_{E}(O, X)=\left(e^{r-b}-1\right) /\left(e^{r-b}+1\right) .
$$

Let $Q \in S_{P}(b)$ be any point and let $Y$ be the intersection of the circle $S_{O}(r-b)$ and the line segment $O Q$. Now, because the hyperbolic metric "grows" with the distance from $O$ it is easy to see that the intrinsic length of the piece of the 
circle $S_{O}(r-b)$ joining $X$ and $Y$ is smaller than $\operatorname{dist}_{H}(P, Q)=b$. So if we denote the arc joining $X$ and $Y$ by $\operatorname{arc}(X, Y)$ we have from (1.4)

$$
\begin{aligned}
\operatorname{length}_{E}(\operatorname{arc}(X, Y)) & <\frac{1}{2} \cdot\left(1-\operatorname{dist}_{E}(O, X)\right) \cdot \operatorname{length}_{H}(\operatorname{arc}(X, Y)) \\
& <\frac{1}{2} \cdot\left(1-\frac{e^{r-b}-1}{e^{r-b}+1}\right) \cdot b<\frac{b}{2} \cdot e^{-(r-b)} .
\end{aligned}
$$

Now, for the $\varphi \angle(O P, O Q)=\angle(O X, O Y)$, we have

$$
\varphi<b e^{-(r-b)} / 2, \quad\left(\text { because } \operatorname{dist}_{E}(O, X)<1\right)
$$

which proves the lemma.

Returning to our convex set $C=C(v, \alpha, r), v \in T_{P} N^{n}$, define

$$
\begin{aligned}
& \beta=\sup \left\{\angle(v, \overrightarrow{P Q}): Q \in S^{n-1}(\infty) \backslash \bar{C}\right\} \\
& \beta_{i}=\sup \left\{\angle(P X, P Y): X \in T_{i}, \quad Y \in T_{i+1}, \operatorname{dist}(X, Y)=1\right\}
\end{aligned}
$$

From the construction of $C$ it is now obvious that

$$
\beta \leq \alpha+\sum_{i} \beta_{i}
$$

According to Lemma 1.2.

$$
\beta_{i}<\frac{e}{2} e^{-r_{i}}
$$

so we have for $\beta$

$$
\beta<\alpha+\frac{e}{2} \cdot \sum_{i} e^{-r_{i}}
$$

To prove that $C(v, \alpha, r)$ contains a cone, we have to show that if we choose $r$ large enough then $\beta<\pi$; that is, we have to prove that

$$
\sum_{i} e^{-r_{i}} \rightarrow 0 \text { as } r \rightarrow \infty
$$

This will be done in the next section.

\section{Solution OF THE Dirichlet PROBlem}

First we prove the existence of arbitrarily "small" convex neighborhoods of $X \in S^{n-1}(\infty)$.

Theorem 2.1. Suppose $N^{n}$ satisfies the conditions of Theorem 0.1 . Let $O \in N^{n}$. Given any $v \in T_{O} N^{n},|v|=1, \delta>0, q>0$ there is a convex set $C$, such that

(i) $C \subset T(v, \delta, q)$,

(ii) $T\left(v, \delta^{\prime}\right) \cap S^{n-1}(\infty) \subset \bar{C} \cap S^{n-1}(\infty)$ for some small $0<\delta^{\prime}<\delta$.

Proof. Let $\gamma$ be the geodesic from $O$ such that $\gamma^{\prime}(0)=v$ and set $\alpha=3 \pi / 4$. Construct according to $\S 1$ the convex set $C=C\left(-\gamma^{\prime}(t), \alpha, s t\right)$ for some $0<$ $s<1$.

First we prove (ii). Because the cone topology of $\bar{N}^{n}$, defined at the beginning of $\S 1$, does not depend on the base point (see [5]), it is enough to prove that, 
for some large $t, C$ contains a cone, that is $C \supset T\left(\gamma^{\prime}(t), \eta\right)$ for some $\eta>0$. That is, using the notation of $\S 1$, we have to prove that

$$
\sum_{i} \beta_{i} \rightarrow 0 \text { as } r \rightarrow \infty
$$

where, according to the construction of $C, r=r_{0}=s t, r_{n}=r_{n-1}+\varepsilon_{n-1}$. Recall the definition of $\varepsilon_{n-1}$ from Proposition 1.1:

$$
\varepsilon_{n}=\delta / a_{n},
$$

where $\delta>0$ is an absolute constant and

$$
a_{n}=\max \left\{|K(\alpha)|: \alpha \text { is a two-plane at } R \in B_{P}\left(r_{n}+1\right)\right\} \text {. }
$$

Because $a_{n} \leq g\left(t+r_{n}+1\right)$, where $g$ is the function from Theorem 0.1 , we can choose

$$
\varepsilon_{n}=\delta / g\left(t+r_{n}+1\right)
$$

and use this $\varepsilon_{n}$ in the construction of the convex set $C$. It is obvious that the sequence $r_{n} \rightarrow \infty$. Otherwise, if $r_{n}<L$ then $\varepsilon_{n}$ would be bounded away from 0 which would lead to a contradiction.

Denote by $t_{n}$ the number of $r_{i}$ 's in the interval $\left[2^{n} r, 2^{n+1} r\right], n=0,1,2, \ldots$ and let

$$
\omega_{n}=\sum_{2^{n} r \leq r_{i} \leq 2^{n+1} r} \beta_{i}
$$

Using (1.6) we have

$$
\omega_{n}<t_{n} \cdot \frac{e}{2} \cdot e^{-2^{n} r} .
$$

The function $g$ is nondecreasing, therefore, $\varepsilon_{n}$ is nonincreasing and hence we have the following

$$
t_{n} \cdot \delta / g\left(t+2^{n+1} r+1\right)<2^{n} r .
$$

Combining the two inequalities we have

$$
\omega_{n}<\frac{e}{2 \delta} \cdot 2^{n} r \cdot g\left(t+2^{n+1} r+1\right) \cdot e^{-2^{n} r} .
$$

By the assumption of Theorem 0.1 we know that $g(t)<e^{\lambda t}$ for large enough $t$. Recalling that $r=s t$ we have

$$
\omega_{n}<\frac{e^{1+\lambda}}{2 \delta} \cdot 2^{n} s t \cdot e^{-2^{n} s t} \cdot e^{\lambda\left(t+2^{n+1} s t\right)} .
$$

We can write the exponent of $e$ in the form of $t\left(\lambda+(2 \lambda-1) 2^{n} s\right)$. Because $\lambda<1 / 3$ we can choose $s$ sufficiently close to 1 such that

$$
\lambda+(2 \lambda-1) 2^{n} s=c_{n}<0, \quad \text { for } n=0,1,2, \ldots .
$$

Moreover it is easy to see that $c_{n}$ decreases rapidly, that is

$$
c_{n}-c_{n+1}=-2(2 \lambda-1) \cdot 2^{n} s>-2(2 \lambda-1) s=d>0 \text {. }
$$

Now we have, with an absolute constant $L$, for $\omega_{n}$

$$
\omega_{n}<L 2^{n} t \cdot e^{t\left(c_{0}-n d\right)}<L \cdot e^{(t / 2) c_{0}-n(t d-1)},
$$

for large enough $t$. Because $c_{0}<0$ and $d>0$ it is easy to see that

$$
\sum_{i} \beta_{i}<\sum_{i} \omega_{i}<L \cdot e^{(t / 2) c_{0}} \cdot \sum_{i} e^{-i(t d-1)} \rightarrow 0 \text { as } t \rightarrow \infty .
$$

That proves (2.1) and claim (ii) as well.

To prove (i) we need the following fact: 
Fact. If $N^{n}$ satisfies the condition of Theorem 2.1 then there is a $t_{0}$, depending on $q, \delta, \alpha$, such that for any $t>t_{0}$ we have $T\left(\gamma^{\prime}(t), \pi-\alpha\right) \subset T(v, \delta, q)$.

Proof of Fact. Consider the geodesic triangle $O \gamma(t) Q$ in $N^{n}$, where $\angle(\gamma(t) O, \gamma(t) Q)=\alpha, \operatorname{dist}_{N}(\gamma(t), Q)=p$. Because the above statement is obviously true in $H^{2}(-1)$ we only have to prove that

$$
\varphi=\angle(O \gamma(t), O Q) \leq \tilde{\varphi}=\angle(\widetilde{O} \tilde{\gamma}(t), \tilde{O} \widetilde{Q})
$$

where $\tilde{O} \tilde{\gamma}(t) \widetilde{Q}$ is the corresponding geodesic triangle in $H^{2}(-1)$. It now follows from the Rauch comparison theorem (see [3,1.28 and 1.30]) that $u=$ $\operatorname{dist}_{N}(O, Q) \geq \operatorname{dist}_{H}(\widetilde{O}, \widetilde{Q})=\tilde{u}$. Form the geodesic triangle $\widetilde{O} \tilde{\gamma}(t) \widetilde{Q}_{1}$ in $H^{2}(-1)$, such that $\operatorname{dist}_{H}\left(\widetilde{O}, \widetilde{Q}_{1}\right)=u$, $\operatorname{dist}_{H}(\widetilde{O}, \tilde{\gamma}(t))=t$, and $\operatorname{dist}_{H}\left(\tilde{\gamma}(t), \widetilde{Q}_{1}\right)$ $=p$. Then $\widetilde{Q}_{1}$ is in the intersection of the circles $S_{\widetilde{o}}(u)$ and $S_{\tilde{\gamma}(t)}(p)$. In $H^{2}(-1)$ geodesic balls are actually Euclidean balls (because isometries of $H^{2}(-1)$ are the Moebius transformations that preserve circles, but not centers) and because $u \geq \tilde{u}$ it is fairly easy to see that

$$
\angle\left(\widetilde{O} \tilde{\gamma}(t), \widetilde{O} \widetilde{Q}_{1}\right) \leq \tilde{\varphi} .
$$

Again applying Topogonov's theorem [7, Theorem 2.7.6] to the geodesic triangles $O \gamma(t) Q$ and $\widetilde{O} \tilde{\gamma}(t) \widetilde{Q}_{1}$, we see that

$$
\varphi=\angle(O \gamma(t), O Q) \leq \angle\left(\tilde{O} \tilde{\gamma}(t), \tilde{O} \widetilde{Q}_{1}\right) \leq \angle(\tilde{O} \tilde{\gamma}(t), \tilde{O} \tilde{Q})=\tilde{\varphi}
$$

This proves (2.2) and the fact as well.

We now return to the proof of Theorem 2.1. From the construction of $C=$ $C\left(-\gamma^{\prime}(t), \alpha, s t\right)$ it is obvious that

$$
C \subset B_{\gamma(t)}(s t) \cup T\left(\gamma^{\prime}(t), \pi-\alpha\right) .
$$

Fact implies that for large $t, T\left(\gamma^{\prime}(t), \pi-\alpha\right) \subset T(v, \delta, q)$ and from Lemma 1.2 it is obvious that for large $t, B_{\gamma(s)}(s t) \subset T(v, \delta, q)$. This proves (i) and the theorem as well.

Proof of Theorem 0.1. As a consequence of Theorem 2.1. and Choi's theorem (Theorem A) we have our main theorem. The proof is rather easy moreover it is exactly the same as of Theorem 3.2. in [1].

Because the curvature $K \leq-1, N^{n}$ satisfies the visibility property (see [5]), that is, for each $x, y \in S^{n-1}(\infty)$ there is a unique geodesic "joining" $x$ and $y$ (being in the asymptote class defined by $x$ and $y$ as in $\S 1$ ). Therefore we can define the convex hull $C(S)$ of a given set $S \subset S^{n-1}(\infty)$ to be the smallest geodesically closed set in $\bar{N}^{n}$ containing $S$.

The main tool in the proof of [1, Theorem 3.3] was the existence of "large" convex sets, that is, the following:

Lemma 2.2. Suppose $N^{n}$ satisfies the assumptions of Theorem 0.1 . Then for each $O \in N^{n}$ and $v \in T_{O} N^{n}$ and for any $0<\alpha<\gamma<\pi$, for large enough $t$ the convex set $C=C(v, \alpha, t)$ will satisfy

$$
S^{n-1}(\infty) \cap T(-v, \pi-\gamma) \subset \bar{C} \cap S^{n-1}(\infty) \subset S^{n-1}(\infty) \cap T(-v, \pi-\alpha) .
$$


Proving Lemma 2.2 is the same as proving (1.7) or (2.1) which was basically done in the proof of claim (ii) in the proof of Theorem 2.1 .

Now the proof of [1, Theorem 3.3] applies without any change in our case too. So we have the analogous theorem.

Theorem 2.3. Let $N^{n}$ be a complete simply connected Riemannian manifold satisfying the assumptions of Theorem 0.1 . If $S$ is a closed set in $S^{n-1}(\infty)$, then $C(S) \cap S^{n-1}(\infty)=S$.

\section{REFERENCES}

1. M. T. Anderson, The Dirichlet problem at infinity for manifolds of negative curvature, J. Differential Geom. 18 (1983), 701-721.

2. W. Ballmann, On the Dirichlet problem at infnity for manifolds of nonpositive curvature, Forum Math. 1 (1989), 201-213.

3. J. Cheeger and D. Ebin, Comparison theorems in Riemannian geometry, North-Holland, Amsterdam, 1975.

4. H. I. Choi, Asymptotic Dirichlet problems for harmonic functions on Riemannian manifolds, Thesis, Univ. California, Berkeley, 1982.

5. P. Eberlein and B. O'Neill, Visibility manifolds, Pacific J. Math. 46 (1973), 45-109.

6. W. I. Kifer, Brownian motion and harmonic functions on manifolds of negative curvature, Theory of Probab. Appl. 21 (1976), 81-95.

7. W. Klingenberg, Riemannian geometry, De Gruyter, 1982.

8. D. Sullivan, The Dirichlet problem at infinity for a negatively curved manifold, J. Differential Geom. 18 (1983), 723-732.

Department of Mathematics, University of Notre Dame, Notre Dame, Indiana 46556 